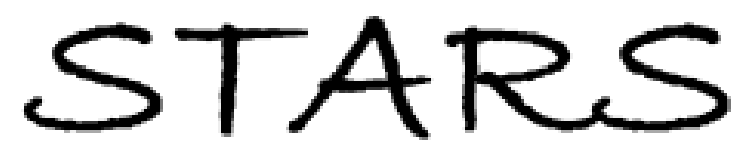

University of Central Florida

STARS

$1-1-2010$

\title{
Reconstruction of Planar Conductivities In Subdomains From Incomplete Data
}

Adrian Nachman

Alexandru Tamasan

University of Central Florida

Alexandre Timonov

Find similar works at: https://stars.library.ucf.edu/facultybib2010

University of Central Florida Libraries http://library.ucf.edu

This Article is brought to you for free and open access by the Faculty Bibliography at STARS. It has been accepted for inclusion in Faculty Bibliography 2010 s by an authorized administrator of STARS. For more information, please contact STARS@ucf.edu.

\section{Recommended Citation}

Nachman, Adrian; Tamasan, Alexandru; and Timonov, Alexandre, "Reconstruction of Planar Conductivities In Subdomains From Incomplete Data" (2010). Faculty Bibliography 2010s. 576.

https://stars.library.ucf.edu/facultybib2010/576

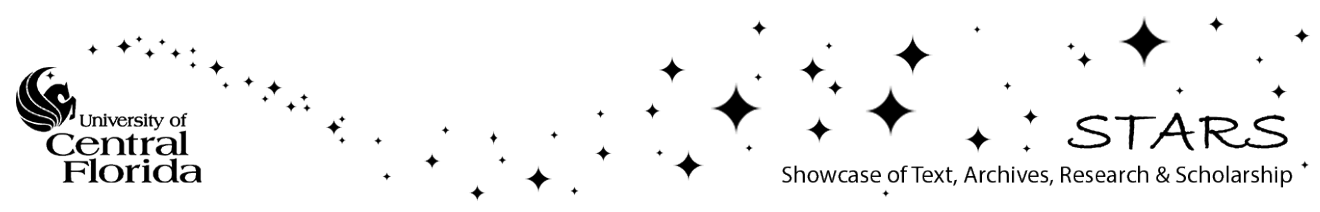




\title{
RECONSTRUCTION OF PLANAR CONDUCTIVITIES IN SUBDOMAINS FROM INCOMPLETE DATA*
}

\author{
ADRIAN NACHMAN ${ }^{\dagger}$, ALEXANDRU TAMASAN $\ddagger$, AND ALEXANDRE TIMONOV
}

\begin{abstract}
We consider the problem of recovering a sufficiently smooth isotropic conductivity from interior knowledge of the magnitude of the current density field $|J|$ generated by an imposed voltage potential $f$ on the boundary. In any dimension $n \geq 2$, we show that equipotential sets are global area minimizers in the conformal metric determined by $|J|$. In two dimensions, assuming the boundary voltage is almost two-to-one, we prove uniqueness of the minimization problem. This yields two results on reconstruction from incomplete data. In the first case, $|J|$ is known in all of $\Omega$, but the almost two-to-one $f$ is know only on subintervals of the boundary. The second case assumes that $|J|$ is known only in an appropriate subdomain $\tilde{\Omega}$ : our method works provided that $\tilde{\Omega}$ contains entire equipotential curves joining boundary points. Based on solving two point boundary value problems for the geodesic system, we give a procedure to determine whether $\tilde{\Omega}$ satisfies this property, to construct the equipotential curves lying entirely in the interior of $\tilde{\Omega}$, and to obtain the conductivity in the region spanned by these curves. We also conduct a numerical study to illustrate the computational feasibility of the method.
\end{abstract}

Key words. conductivity imaging, current density impedance imaging, minimal surfaces, 1Laplacian

AMS subject classifications. 35R30, 35J60, 31A25, 62P10

DOI. $10.1137 / 10079241 \mathrm{X}$

1. Introduction. We consider the problem of reconstructing an isotropic conductivity $\sigma$ when knowledge of the magnitude of one current density $|J|$ generated by an imposed voltage $f$ on the boundary is only partially available. The voltage potential $u$ is the unique solution of the Dirichlet problem for the conductivity equation

$$
\nabla \cdot \sigma \nabla u=0,\left.\quad u\right|_{\partial \Omega}=f
$$

and the current density field is defined by Ohm's law: $J=-\sigma \nabla u$. Currently, interior knowledge of $J$ is obtained from magnetic resonance measurements as discovered in [27], but recent engineering advances show that magneto-acoustic measurements in biological tissue can also recover $J[29]$.

The classical problem of electrical impedance tomography is exponentially illposed, yielding images of low resolution away from the boundary [22], [9]. In an earlier paper [23] we have shown that knowledge of one $|J|$ in the interior yields stable reconstruction and increases both the quantitative accuracy and the resolution. In this paper, for two dimensional models, we show that such a reconstruction is local, in the sense that knowledge of $|J|$ in an appropriate subregion yields the conductivity in that subregion. We will describe below precisely what subregions are appropriate.

*Received by the editors April 15, 2010; accepted for publication (in revised form) October 5, 2010; published electronically December 22, 2010.

http://www.siam.org/journals/siap/70-8/79241.html

$\dagger$ Department of Mathematics and Edward S. Roger Sr. Department of Electrical and Computer Engineering, University of Toronto, Toronto, ON M5S 2E4, Canada (nachman@math.toronto.edu). This author's work was supported by an NSERC Discovery Grant.

${ }^{\ddagger}$ Department of Mathematics, University of Central Florida, Orlando, FL 32816 (tamasan@math. ucf.edu). This author's work was supported by NSF grant DMS-0905799.

$\S$ Division of Mathematics and Computer Science, University of South Carolina Upstate, Spartanburg, SC 29303 (atimonov@uscupstate.edu). 
Among the first works which considered interior data in conductivity imaging, we mention [30], [14], [15], [16], [13], and [18]. The papers [20] and [19] use the interior knowledge of one component of the magnetic field; see [28] for comprehensive references in this direction. Recent work combines electrical impedance tomography with ultrasound, electro-acoustic measurements, or magneto-acoustic measurements; see $[21],[3],[2],[10],[4]$.

In [23], we first proposed a reconstruction method which uses the interior knowledge of the magnitude of one current density field $|J|$. The reconstruction was based on the fact that, in the absence of singularities, equipotential sets have zero mean curvature (i.e., are minimal surfaces) in the metric $g=|J|^{2 /(n-1)} I$ conformal to the Euclidean metric; $n$ is the dimension of the domain. The equipotential surfaces are critical points for the functional

$$
\mathbf{E}(\Sigma)=\int_{\Sigma}|J| d S
$$

where $d S$ is the induced Euclidean surface measure. Note that $\mathbf{E}(\Sigma)$ is the area of $\Sigma$ in the Riemannian metric $g$ described above.

In section 2 of this paper (Theorem 2.6) we prove a stronger result: equipotential sets are in fact minimizers of $\mathbf{E}(\Sigma)$. We stress that $\mathbf{E}$ in (1.2) is not convex in $\Sigma$.

If the interior data $|J|$ is known throughout $\Omega$, and $f$ is known on the entire boundary, then, as the authors show in [24], the voltage potential $u$ is a minimizer of the functional

$$
\int_{\Omega}|J| \cdot|\nabla v| d x
$$

subject to $v \in W^{1,1}(\Omega)$ with $v=f$ at the boundary, $\partial \Omega$. Moreover, $u$ is the unique minimizer among $v \in W^{1,1}(\Omega)$ with $|\nabla v|>0$ a.e. in $\Omega$ and $v=f$ at the boundary, and one can determine it by a minimization algorithm. A structural stability result for the minimization of the functional in (1.3) can be found in [25]. Formally, the Euler-Lagrange equation for the nonsmooth functional in (1.3) is the generalized 1Laplacian. This is in contrast with the work in [3], [2], [10], and [6], where different problems with interior data lead to the generalized 0-Laplacian.

In section 3 we present a new reconstruction method for two dimensional models which yields two new results. In both cases the imposed boundary voltage $f$ is assumed to be almost two-to-one (see [1], [23], or section 3).

In the first result, $|J|$ is known throughout $\Omega$, but $f$ is known only on parts of the boundary. More precisely, if some interval $(\alpha, \beta)$ of boundary voltages is twice contained in the known values of $f$, then we can recover the conductivity in the subregion

$$
\Omega_{\alpha, \beta}:=\{x \in \bar{\Omega}: \alpha<u(x)<\beta\} .
$$

We give a procedure to determine this region from the data.

In the second result, $|J|$ is known only in a subregion $\tilde{\Omega}$. Our method works if $\tilde{\Omega}$ contains regions of the type (1.4) for unknown values $\alpha$ and $\beta$. For example, if $\partial \tilde{\Omega}$ includes the arc of maxima (or minima) of $f$, then this is always the case. We stress, however, that this property of $\tilde{\Omega}$ need not be assumed a priori: the method determines from the data whether $\tilde{\Omega}$ contains regions of the type (1.4), and, if so, recovers all the (maximal) intervals $(\alpha, \beta)$, their corresponding $\Omega_{\alpha, \beta}$, and the conductivity therein. 
If $\tilde{\Omega}$ does not contain regions of type (1.4), then the incomplete interior data is insufficient for recovering the conductivity in any subregion. As in the first case, we use only the boundary voltages that are twice contained in the known values of $f$; see Theorem 3.4 for the uniqueness results and Theorem 3.6 for the reconstruction of one equipotential line. Our reconstruction method from incomplete data is described following the proof of Theorem 3.6.

Note that, for almost two-to-one boundary voltages, not only are the equipotential curves globally length minimizing (Theorem 2.6), but they are unique with this property (see the proof of Theorem 3.4). This unique minimizing property is preserved if we restrict the competitors to curves contained in $\tilde{\Omega}$.

In section 3.1 we give a reconstruction method from the partial data discussed above, based on the computation of the geodesics within $\tilde{\Omega}$ that join pairs of equipotential points at the boundary.

In section 4 we present a numerical study to test the computational feasibility of the reconstruction method. The algorithm is based on the numerical solution of the two point boundary value problem for the geodesic system.

2. Equipotential surfaces are area minimizing in the conformal metric. In this section we prove that, in any dimension $n \geq 2$, the equipotential sets are global minima of $\mathbf{E}(\Sigma)$. This result is a consequence of the minimizing property of the voltage potential for the functional (1.3) first established in [24]. The proof adapts the approach of Bombieri, De Giorgi, and Giusti [8].

We start by recalling the co-area formula; see, e.g., [12], [31].

Theorem 2.1 (co-area formula). Let $u \in \operatorname{Lip}(\Omega)$, and let a be integrable in $\Omega \subset \mathbb{R}^{n}$. Then, for a.e. $t \in \mathbb{R}, H^{n-1}\left(u^{-1}(t) \cap \Omega\right)<\infty$ and

$$
\int_{\Omega} a|\nabla u(x)| d x=\int_{-\infty}^{\infty} \int_{u^{-1}(t)} a d H^{n-1}(x) d t
$$

where $H^{n-1}$ is the $(n-1)$ dimensional Hausdorff measure.

Note that, since $H^{n-1}\left(u^{-1}(t) \cap \Omega\right)<\infty$, we also have $H^{n}\left(u^{-1}(t) \cap \Omega\right)=0$ for a.e. $t \in \mathbb{R}$.

Proposition 2.2. Let $a \geq 0$ be integrable in $\Omega$, and let

$$
u \in \operatorname{argmin}\left\{\int_{\Omega} a|\nabla v| d x: \quad v \in \operatorname{Lip}(\Omega),\left.\quad v\right|_{\partial \Omega}=f\right\}
$$

For $\lambda \in \mathbb{R}$ arbitrarily fixed, let $u_{+}=\max \{u-\lambda, 0\}$ and $u_{-}=\min \{u, \lambda\}$ be defined in $\Omega$, and let $f_{+}=\max \{f-\lambda, 0\}$, respectively, $f_{-}=\min \{f, \lambda\}$, be defined on the boundary $\partial \Omega$. Then

$$
\begin{aligned}
& u_{+} \in \operatorname{argmin}\left\{\int_{\Omega} a|\nabla v| d x: \quad v \in \operatorname{Lip}(\Omega),\left.\quad v\right|_{\partial \Omega}=f_{+}\right\}, \\
& u_{-} \in \operatorname{argmin}\left\{\int_{\Omega} a|\nabla v| d x: \quad v \in \operatorname{Lip}(\Omega),\left.\quad v\right|_{\partial \Omega}=f_{-}\right\} .
\end{aligned}
$$

Copyright $@$ by SIAM. Unauthorized reproduction of this article is prohibited. 
Proof. Note that $u_{ \pm} \in \operatorname{Lip}(\Omega)$ and $u=u_{+}+u_{-}$. Using the co-area formula (2.1) we have

$$
\begin{aligned}
\int_{\Omega} a|\nabla u| d x & =\int_{-\infty}^{\lambda} \int_{u^{-1}(t)} a d H^{n-1}(x) d t+\int_{\lambda}^{\infty} \int_{u^{-1}(t)} a d H^{n-1}(x) d t \\
& =\int_{-\infty}^{\lambda} \int_{\left(u_{-}\right)^{-1}(t)} a d H^{n-1}(x) d t+\int_{0}^{\infty} \int_{\left(u_{+}\right)^{-1}(t)} a d H^{n-1}(x) d t \\
& =\int_{-\infty}^{\infty} \int_{\left(u_{-}\right)^{-1}(t)} a d H^{n-1}(x) d t+\int_{-\infty}^{\infty} \int_{\left(u_{+}\right)^{-1}(t)} a d H^{n-1}(x) d t \\
& =\int_{\Omega} a\left|\nabla u_{-}\right| d x+\int_{\Omega} a\left|\nabla u_{+}\right| d x
\end{aligned}
$$

To prove (2.3), we observe that for any $w \in \operatorname{Lip}(\Omega)$ with $w=0$ on the boundary, the following estimate holds:

$$
\begin{gathered}
\int_{\Omega} a\left|\nabla u_{+}\right| d x=\int_{\Omega} a|\nabla u| d x-\int_{\Omega} a\left|\nabla u_{-}\right| d x \leq \int_{\Omega} a|\nabla(u+w)| d x-\int_{\Omega} a\left|\nabla u_{-}\right| d x \\
=\int_{\Omega} a\left|\nabla\left(u_{+}+u_{-}+w\right)\right| d x-\int_{\Omega} a\left|\nabla u_{-}\right| d x \leq \int_{\Omega} a\left|\nabla\left(u_{+}+w\right)\right| d x .
\end{gathered}
$$

The first inequality in the above uses (2.2), while the second is the triangle inequality.

The minimizing property of $u_{-}$in (2.4) can be shown similarly.

Corollary 2.3. Let $a \geq 0$ be integrable in $\Omega$ and $u \in \operatorname{Lip}(\Omega)$ such that

$$
u \in \operatorname{argmin}\left\{\int_{\Omega} a|\nabla v| d x: \quad v \in \operatorname{Lip}(\Omega),\left.\quad v\right|_{\partial \Omega}=f\right\} .
$$

For every $\lambda \in \mathbb{R}$ and $\epsilon>0$ define

$$
u_{\lambda, \epsilon}:=\frac{1}{\epsilon} \min \{\epsilon, \max \{u-\lambda, 0\}\}
$$

and let $f_{\lambda, \epsilon}$ be its trace on the boundary $\partial \Omega$.

Then $u_{\lambda, \epsilon} \in \operatorname{Lip}(\Omega)$ and

$$
u_{\lambda, \epsilon} \in \operatorname{argmin}\left\{\int_{\Omega} a|\nabla v| d x: \quad v \in \operatorname{Lip}(\Omega),\left.\quad v\right|_{\partial \Omega}=f_{\lambda, \epsilon}\right\} .
$$

Proof. This is a direct consequence of Proposition 2.2 applied twice.

Lemma 2.4. Let $a, u \in \operatorname{Lip}(\Omega)$ with $|\nabla u|>0$ a.e. in $\Omega$ and

$$
\nabla u /|\nabla u| \in W^{1,1}(\Omega) .
$$

Then, for almost every $\lambda \in \operatorname{Range}(u)$,

$$
\lim _{\epsilon \rightarrow 0^{+}} \int_{\Omega} a\left|\nabla u_{\lambda, \epsilon}\right| d x=\int_{u^{-1}(\lambda)} a d H^{n-1}(x)
$$

with $u_{\lambda, \epsilon}$ as defined in (2.6).

Proof. From Theorem 2.1, we get that $H^{n-1}\left(u^{-1}(\lambda) \cap \Omega\right)<\infty$ a.e. $\lambda \in \mathbb{R}$. In particular, for a.e. $\lambda \in \mathbb{R}$,

$$
H^{n}\left(u^{-1}(\lambda) \cap \Omega\right)=0
$$

Copyright (c) by SIAM. Unauthorized reproduction of this article is prohibited. 
Note that $u \in \operatorname{Lip}(\Omega)$ extends continuously to the boundary. Since $H^{n-1}(\partial \Omega)<\infty$, from the disjoint partition $\partial \Omega=\cup_{\lambda \in R}\left(u^{-1}(\lambda) \cap \partial \Omega\right)$, we have $H^{n-1}\left(u^{-1}(\lambda) \cap \partial \Omega\right)>0$ for at most countable many $\lambda$ 's. In particular, for a.e. $\lambda \in \mathbb{R}$,

$$
H^{n-1}\left(u^{-1}(\lambda) \cap \partial \Omega\right)=0 .
$$

Let $\lambda \in$ Range $(u)$ be such that both (2.10) and (2.11) hold, and let $\epsilon>0$. Recall

$$
u_{\lambda, \epsilon}(x)= \begin{cases}0 & \text { if } u(x)<\lambda, \\ (u(x)-\lambda) / \epsilon & \text { if } \lambda \leq u(x) \leq \lambda+\epsilon, \\ 1 & \text { if } u(x)>\lambda+\epsilon .\end{cases}
$$

From the co-area formula (2.1) we have

$$
\begin{aligned}
\int_{\Omega} a\left|\nabla u_{\lambda, \epsilon}\right| d x & =\int_{-\infty}^{\infty} \int_{\left(u_{\lambda, \epsilon}\right)^{-1}(t)} a d H^{n-1}(x) d t \\
& =\int_{0}^{1} \int_{\{x: u(x)=\lambda+t \epsilon\}} a d H^{n-1}(x) d t .
\end{aligned}
$$

To finish the proof we show that

$$
\lim _{\epsilon \rightarrow 0} \int_{\{x: u(x)=\lambda+t \epsilon\}} a d H^{n-1}(x)=\int_{\{x: u(x)=\lambda\}} a d H^{n-1}(x)
$$

holds uniformly a.e. $t \in[0,1]$. The domain $\Omega_{t \epsilon}:=\{x \in \Omega: \lambda<u(x)<\lambda+t \epsilon\}$ is Lipschitz. Since $a \in \operatorname{Lip}(\Omega)$, it extends continuously to the boundary, and, using (2.8), $a \nabla u /|\nabla u| \in W^{1,1}(\Omega)$ extends to the boundary $\partial \Omega$ as a bounded function. From (2.8) we also have $\nabla \cdot(a \nabla u /|\nabla u|) \in L^{1}(\Omega)$. Let $\nu$ denote the outer unit normal to the boundary. Then the Green's formula in $\Omega_{t \epsilon}$ yields

$$
\begin{aligned}
\left|\int_{u^{-1}(\lambda+t \epsilon)} a d H^{n-1}-\int_{u^{-1}(\lambda)} a d H^{n-1}\right| \leq & \left|\int_{\{x \in \partial \Omega: \lambda<u(x)<\lambda+\epsilon\}} a \frac{\nabla u}{|\nabla u|} \nu d H^{n-1}(x)\right| \\
& +\left|\int_{\{x \in \Omega: \lambda<u(x)<\lambda+\epsilon\}} \nabla \cdot a \frac{\nabla u}{|\nabla u|} d x\right| .
\end{aligned}
$$

Using (2.10) we have

$$
\begin{aligned}
\lim _{\epsilon \rightarrow 0} H^{n}(\{x \in \Omega: \lambda<u(x)<\lambda+\epsilon\}) & =H^{n}\left(\bigcap_{\epsilon>0}\{x \in \Omega: \lambda<u(x)<\lambda+\epsilon\}\right) \\
& \leq H^{n}\left(\bigcap_{\epsilon>0}\{x \in \Omega: \lambda \leq u(x)<\lambda+\epsilon\}\right) \\
& =H^{n}\left(u^{-1}(\lambda) \cap \Omega\right)=0 .
\end{aligned}
$$

Similarly, using (2.11), we obtain

$$
\lim _{\epsilon \rightarrow 0} H^{n-1}(\{x \in \partial \Omega: \lambda<u(x)<\lambda+\epsilon\})=0 .
$$

This proves (2.13). By taking the limit with $\epsilon \rightarrow 0$ in (2.12) and using (2.13), we obtain (2.9). 
Corollary 2.5. Let $\lambda \in \mathbb{R}$ satisfy (2.11) and (2.10). Then the limit (2.9) holds for such $a \lambda$.

We remark that Lemma 2.4 holds for a bounded continuous coefficient $a$ and without the assumption (2.8) in $u$. In such a case,

$$
\lim _{\epsilon \rightarrow 0^{+}} \int_{\Omega} a\left|\nabla u_{\lambda, \epsilon}\right| d x=\left\|D \chi_{\lambda}\right\|(a)
$$

where the right-hand side is the total variation of the indicator function $\chi_{\lambda}$ of the superlevel set $\{x: u(x)>\lambda\}$, interpreted as a Radon measure applied to the continuous map $a$. However, the extension (2.14) is beyond the scope of this paper.

THEOREM 2.6 (minimizing property of level sets). Let $\Omega \subset \mathbb{R}^{n}, n \geq 2$, be a domain with Lipschitz boundary. Assume $\sigma \in C^{1, \delta}(\Omega), 0<\delta<1$, is bounded away from zero and $f \in C^{2, \delta}(\partial \Omega)$. Let $u \in C^{2, \delta}(\bar{\Omega})$ be the solution of (1.1), and let $|J|=|\sigma \nabla u|$. Assume that $|J|>0$ in $\bar{\Omega}$.

Then, for a.e. $\lambda \in \mathbb{R}$ and for any $v \in C^{2}(\bar{\Omega})$ with $v=f$ on $\partial \Omega$ and $\min _{\bar{\Omega}}|\nabla v|>0$,

$$
\mathbf{E}\left(u^{-1}(\lambda)\right) \leq \mathbf{E}\left(v^{-1}(\lambda)\right)
$$

Furthermore, if $f$ satisfies (2.11) for every $\lambda$, then (2.15) holds for all $\lambda \in \mathbb{R}$.

Proof. For $\lambda \notin \operatorname{Range}(u)$, the left-hand side of (2.15) is zero and the inequality is trivial. Since $u$ obeys the maximum principle and coincides with $v$ at the boundary, Range $(u) \subset$ Range $(v)$.

Now let $\lambda \in \operatorname{Range}(u)$ be arbitrary. Since both $u$ and $v$ are free of singular points, their corresponding $\lambda$-level set is a $C^{2}$-smooth oriented surface. In particular the $H^{n-1}$-measure coincides with the induced Lebesgue measure on the respective surface. Moreover, $u$ and $v$ satisfy (2.10), and, by assumption, (2.11).

Since $u$ is a solution of (1.1), by [24, Proposition 1.2],

$$
u \in \operatorname{argmin}\left\{\int_{\Omega} a|\nabla v| d x: \quad v \in \operatorname{Lip}(\Omega),\left.\quad v\right|_{\partial \Omega}=\left.u\right|_{\partial \Omega}\right\} .
$$

For $\epsilon>0$ arbitrarily fixed, let $u_{\lambda, \epsilon}$ be defined by (2.6), and define similarly $v_{\lambda, \epsilon}:=\min \{\epsilon, \max \{v-\lambda, 0\}\} / \epsilon$. Since $v=u$ coincide at the boundary, we also have $u_{\lambda, \epsilon}=v_{\lambda, \epsilon}$ on $\partial \Omega$.

From Corollary 2.3 we have

$$
\int_{\Omega} a\left|\nabla u_{\lambda, \epsilon}\right| d x \leq \int_{\Omega} a\left|\nabla v_{\lambda, \epsilon}\right| d x
$$

Since $u, v \in C^{2}(\bar{\Omega})$ and their corresponding gradient is bounded away from zero, then $\nabla u /|\nabla u|, \nabla v /|\nabla v| \in C^{1}(\bar{\Omega})$, and (2.8) is satisfied.

Now use Corollary 2.5 to take the limit with $\epsilon \rightarrow 0$ in (2.16) and conclude (2.15).

3. Partial determination of planar conductivities. In this section we show uniqueness of the minimizer of (1.2) for smooth curves in a simply connected planar domain with smooth boundary. The imposed boundary voltage is assumed to be almost two-to-one, i.e., a continuous function whose set of local maxima is one connected arc (that may reduce to one point). This ensures that $|\nabla u| \neq 0$ in $\Omega[1]$, $[16],[23]$.

We start by recalling some definitions and results from [23] and [24].

Solutions $u$ of the conductivity equation in (1.1) are called $\sigma$-harmonic functions. 
Definition 3.1 (see [24]). A pair of functions $(f, a) \in H^{1 / 2}(\partial \Omega) \times L^{2}(\Omega)$ is called admissible if there exists a positive map $\sigma \in L^{\infty}(\Omega)$ bounded away from zero such that if $u \in H^{1}(\Omega)$ is the $\sigma$-harmonic map with $\left.u\right|_{\partial \Omega}=f$, then

$$
|\sigma \nabla u|=a .
$$

The function $\sigma$ is called a generating conductivity for the pair $(f, a)$, and the function $u$ is called the corresponding potential. The function a is the magnitude of the current in $\Omega$ corresponding to the voltage $f$ on the boundary.

Proposition 3.2 (see [23]). Let $\Omega$ be a simply connected planar domain with $C^{2, \delta}$-boundary, let $\sigma \in C^{1, \delta}(\Omega)$ for some $0<\delta<1$, and let $u$ be $\sigma$-harmonic in $\Omega$ with $\left.u\right|_{\partial \Omega}$ almost two-to-one. Then $|\nabla u|>0$ in $\bar{\Omega}$, and each level set of $u$ is a $C^{2, \delta}$-smooth curve inside $\Omega$ with the two endpoints on the boundary.

The following result is the two dimensional version of Lemma 2.1 in [23]; an alternate proof (specific to two dimensions) is given in the appendix at the end of the paper.

Proposition 3.3. Let $(\Omega, g)$ be a domain in $\mathbb{R}^{2}$, endowed with the metric $g=$ $a^{2} I$ conformal with the Euclidean one, for a given positive factor $a \in C^{1}(\Omega)$. Let $u \in C^{2}(\Omega)$ be such that $|\nabla u|>0$ in $\Omega$. Then the signed curvature of the level curves of $u$ is given by

$$
k=-\frac{1}{a^{2}} \nabla \cdot\left(\frac{a}{|\nabla u|} \nabla u\right) .
$$

The following theorem establishes our main new uniqueness results from incomplete data.

Theorem 3.4 (uniqueness). Let $\Omega \subset \mathbb{R}^{2}$ be a simply connected domain with $C^{3, \delta}$-boundary, $0<\delta<1$. For $i=1,2$ let $\sigma_{i} \in C^{2, \delta}(\Omega)$, let $u_{i}$ be $\sigma_{i}$-harmonic with $\left.u_{i}\right|_{\partial \Omega} \in C^{3, \delta}(\partial \Omega)$ almost two-to-one, and let $\left|J_{i}\right|=\left|\sigma_{i} \nabla u_{i}\right|$. For $\alpha<\beta$ let

$$
\Omega_{\alpha, \beta}:=\left\{x \in \bar{\Omega}: \quad \alpha<u_{1}(x)<\beta\right\} \text { and } \Gamma:=\Omega_{\alpha, \beta} \cap \partial \Omega .
$$

(i) Assume $\left.u_{1}\right|_{\Gamma}=\left.u_{2}\right|_{\Gamma}$ and $\left|J_{1}\right|=\left|J_{2}\right|$ in $\Omega$. Then

$$
\begin{gathered}
u_{1}=u_{2} \text { in } \Omega_{\alpha, \beta} \quad \text { and } \\
\sigma_{1}=\sigma_{2} \text { in } \Omega_{\alpha, \beta} .
\end{gathered}
$$

(ii) Assume $\left.u_{1}\right|_{\Gamma}=\left.u_{2}\right|_{\Gamma}$ and $\left|J_{1}\right|=\left|J_{2}\right|$ in the interior of $\Omega_{\alpha, \beta}$. Then

$$
\begin{gathered}
\left\{x \in \bar{\Omega}: \quad \alpha<u_{2}(x)<\beta\right\}=\Omega_{\alpha, \beta}, \\
u_{1}=u_{2} \text { in } \Omega_{\alpha, \beta}, \quad \text { and } \\
\sigma_{1}=\sigma_{2} \text { in } \Omega_{\alpha, \beta} .
\end{gathered}
$$

Proof. It suffices to prove part (ii). By Proposition 3.2, the equipotential curves are $C^{2, \delta}$-smooth curves in $\Omega$ with the two endpoints on the boundary. From the elliptic regularity theory (see, e.g., [11]), $u \in C^{3, \delta}(\bar{\Omega})$. Therefore, $\left|J_{1}\right|,\left|J_{2}\right| \in C^{2, \delta}(\bar{\Omega})$, and the geodesic system (which involves first order derivatives of the conformal factor) has $C^{1, \delta}(\bar{\Omega})$ right-hand side.

Let $x_{1} \in \Omega_{\alpha, \beta}$, and let $\Sigma_{1}$ be the level set of $u_{1}$ through $x_{1}: \Sigma_{1}=\{x \in \bar{\Omega}$ : $\left.u_{1}(x)=u_{1}\left(x_{1}\right)\right\}$. Clearly $\Sigma_{1} \subset \Omega_{\alpha, \beta}$. Consider now $u_{2}$ along $\Sigma_{1}$. Since $u_{1}=u_{2}$ on $\Gamma$, $u_{2}$ takes the same value at the endpoints $\Sigma_{1} \cap \partial \Omega$. Hence, there is a point $x_{2} \in \Sigma_{1}$ such 
that the derivative of $u_{2}$ at $x_{2}$ in the direction tangential to $\Sigma_{1}$ is zero. If $\Sigma_{2}$ denotes the level set of $u_{2}$ through $x_{2}$ (which is unique by Proposition 3.2), we have that the derivatives of $u_{2}$ at $x_{2}$ in the direction tangential to $\Sigma_{2}$ is also zero. Therefore $\nabla u_{2}\left(x_{2}\right)$ is normal to both $\Sigma_{1}$ and $\Sigma_{2}$, or, equivalently, $\Sigma_{1}$ and $\Sigma_{2}$ are tangent to each other at $x_{2} \in \Omega_{\alpha, \beta}$. Following [23, Theorem 2.1] (see also the appendix) $\Sigma_{1}$ and $\Sigma_{2}$ are both geodesics in $\Omega_{\alpha, \beta}$ endowed with the (same) conformal metric $g=\left|J_{1}\right|^{2} I=\left|J_{2}\right|^{2} I$; $I$ is the Euclidean metric. Since they both pass through $x_{2} \in \Omega_{\alpha, \beta}$ in the same direction, and since the geodesic system has Lipschitz coefficients, $\Sigma_{1}$ and $\Sigma_{2}$ coincide locally near $x_{2}$. Since the entire $\Sigma_{1}$ lies in $\Omega_{\alpha, \beta}$, by the continuation argument for initial value problems of differential equations, $\Sigma_{1}$ and $\Sigma_{2}$ coincide throughout $\Omega_{\alpha, \beta}$. Since $u_{1}$ and $u_{2}$ have the same value at the boundary, $u_{1}=u_{2}$ along $\Sigma_{1}=\Sigma_{2}$; in particular, $\Sigma_{2} \subset \Omega_{\alpha, \beta}$. Since $x_{1}$ has been arbitrarily chosen inside $\Omega_{\alpha, \beta}$, we showed $\Omega_{\alpha, \beta} \subset\left\{x \in \bar{\Omega}: \alpha<u_{2}(x)<\beta\right\}$ and $u_{1}=u_{2}$ in $\Omega_{\alpha, \beta}$.

To prove the reverse inclusion in (3.4), let $x_{\lambda} \in \Omega$ be such that $u_{2}\left(x_{\lambda}\right)=\lambda$, for some fixed $\lambda \in(a, b)$, and let $\Sigma_{\lambda}$ be the level set of $u_{2}$ through $x_{\lambda}$. Since $u_{2}$ already takes the value $\lambda$ at a pair of points in $\Gamma$, and since $u_{2}$ is almost two-to-one at the boundary, $\Sigma_{\lambda} \cap \partial \Omega$ must be in $\Gamma$ and, by the previous argument, coincides with the $\lambda$-level set of $u_{1}$.

Finally, by the choice of almost two-to-one boundary data, $\left|\nabla u_{i}\right| \neq 0(i=1,2)$, and then $\sigma_{1}=|J| /\left|\nabla u_{1}\right|=|J| /\left|\nabla u_{2}\right|=\sigma_{2}$ in $\Omega_{\alpha, \beta}$.

3.1. Determination of equipotential lines. In this section we present a reconstruction method based on the uniqueness result above.

Proposition 3.5. Let $\sigma \in C^{1, \delta}(\Omega)$, and let $u \in C^{2, \delta}(\Omega)$ be $\sigma$-harmonic, with $|\nabla u|>0$ in $\Omega$. Then the level sets of $u$ are geodesics for the metric $g=|J|^{2} I$, where $|J|=|\sigma \nabla u|$. In particular, if $\gamma:(0,1) \rightarrow \Omega, \gamma(t)=(x(t), y(t))$ is any local parametrization of such a level curve, then it satisfies the geodesic system

$$
\begin{gathered}
\ddot{x}=-\dot{x}^{2} \frac{|J|_{x}}{|J|}(x, y)-2 \dot{x} \dot{y} \frac{|J|_{y}}{|J|}(x, y)+\dot{y}^{2} \frac{|J|_{x}}{|J|}(x, y), \\
\ddot{y}=\dot{x}^{2} \frac{|J|_{y}}{|J|}(x, y)-2 \dot{x} \dot{y} \frac{|J|_{x}}{|J|}(x, y)-\dot{y}^{2} \frac{|J|_{y}}{|J|}(x, y),
\end{gathered}
$$

where the dot denotes $\frac{d}{d t}$.

Proof. Since $u \in C^{2}(\Omega)$ with $|\nabla u|>0$, by the implicit function theorem, the level sets of $u$ are $C^{2}$-smooth curves. Since $u$ is $\sigma$-harmonic and $\sigma=|J| /|\nabla u|, u$ solves

$$
\nabla \cdot|J||\nabla u|^{-1} \nabla u=0 .
$$

By (3.2) every level curve of $u$ has constant null curvature; hence it is a geodesic.

The formulae $\Gamma_{i j}^{k}=\frac{1}{2} g^{k l}\left(\partial_{i} g_{j l}+\partial_{j} g_{i l}-\partial_{l} g_{i j}\right)$ compute the Christoffel symbols for the Levi-Civita connection associated with a metric $g$; see, e.g., [17]. In our case $g=|J|^{2} I$, and a simple calculation yields

$$
\Gamma_{11}^{1}=\Gamma_{12}^{2}=-\Gamma_{22}^{1}=\frac{|J|_{x}}{|J|}, \quad \Gamma_{12}^{1}=\Gamma_{22}^{2}=-\Gamma_{11}^{2}=\frac{|J|_{y}}{|J|} .
$$

Using the parametric form for a geodesic curve $\gamma$ (see, e.g., [17]) with the Christoffel symbols in (3.8), we obtain the system (3.7).

Next we show that, under our assumptions, the geodesic system (3.7) subject to two endpoint boundary conditions has a unique solution. Note that this need not be the case in general. 
Theorem 3.6 (reconstruction). Let $(f,|J|) \in C^{2, \delta}(\partial \Omega) \times C^{1, \delta}(\Omega)$ be an admissible pair with $f$ almost two-to-one, and let $\left(x_{0}, y_{0}\right),\left(x_{1}, y_{1}\right) \in \partial \Omega$ be such that $f\left(x_{0}, y_{0}\right)=f\left(x_{1}, y_{1}\right)$. Then the system (3.7), subject to the boundary conditions

$$
(x(0), y(0))=\left(x_{0}, y_{0}\right) \text { and }(x(1), y(1))=\left(x_{1}, y_{1}\right),
$$

has a unique solution $\gamma:[0,1] \rightarrow \Omega, \gamma(t)=(x(t), y(t))$. Moreover, the map $u: \Omega \rightarrow \mathbb{R}$ is constant along $\gamma$ :

$$
(u \circ \gamma)(t)=\lambda, \quad t \in[0,1] .
$$

Proof. Let $\lambda=f\left(x_{0}, y_{0}\right)=f\left(x_{1}, y_{1}\right)$ denote the common voltage. Proposition 3.5 shows that a parametrization (say, by arclength $s$ ) of the level set of $\Sigma=$ $\{(x, y): u(x, y)=\lambda\}$ is a solution of $(3.7)$ which also satisfies $(x(0), y(0))=\left(x_{0}, y_{0}\right)$ and $(x(L), y(L))=\left(x_{1}, y_{1}\right)$, where $L$ is the length (in the metric $g$ ) of $\Sigma$. Since geodesics have constant speed, the scaling $s=L t$ gives a solution to (3.7) which also satisfies (3.9).

We show that the converse also holds; namely, $u$ is constant along any solution of the boundary value problem (3.7), (3.9). Indeed, let $\tilde{\gamma}(t)=(\tilde{x}(t), \tilde{y}(t))$ be a $C^{2}$ solution of (3.7) and (3.9). Since $u \circ \tilde{\gamma}(0)=u \circ \tilde{\gamma}(1)$, there is a point $t_{0} \in(0,1)$, where $\frac{d}{d t}(u \circ \tilde{\gamma})\left(t_{0}\right)=0$. Let $P$ denote the point $\tilde{\gamma}\left(t_{0}\right)$. Now consider the level curve of $u$ passing through $P$, i.e., $\tilde{\Sigma}=\left\{(x, y): u(x, y)=u \circ \tilde{\gamma}\left(t_{0}\right)\right\}$. Since the derivative of $u$ in the direction tangential to $\tilde{\Sigma}$ is also zero, $\tilde{\gamma}$ and $\tilde{\Sigma}$ have the same unit normal at $P$ in the direction of $\nabla u(P)$. Therefore they are tangent to each other at $P$. Since they are both geodesics with respect to the metric $|J|^{2} I$, they coincide throughout $\Omega$.

Since $|\nabla u| \neq 0$, there can be no two level sets intersecting nontangentially. But they must meet at least at the boundary points, and uniqueness follows.

It is important to note that the solution to the problem (3.7), (3.9) depends only on the values of $|J|$ near the curve.

Our reconstruction procedure starts by extending $|J|$ to all of $\Omega$. To find all the equipotential curves which lie entirely in $\tilde{\Omega}$ (the region where $|J|$ is known) we then solve (3.7) subject to (3.9), for each pair of equipotential boundary points. If the solution lies in the interior of $\tilde{\Omega}$, then it is the correct level curve joining those two boundary points. If the calculated curve passes outside $\tilde{\Omega}$ (or touches its boundary) then it is dependent on the extension of $|J|$ and we discard it. An interval $(\alpha, \beta)$ of voltages defines a set $\Omega_{\alpha, \beta}$ as in (1.4) provided that, for each $\lambda \in(\alpha, \beta)$, the calculated $\lambda$-equipotential curve lies entirely in the interior of $\tilde{\Omega}$. If $\tilde{\Omega}$ contains no entire equipotential curves, then all the calculated solutions will be discarded. In such a case the incomplete interior data is insufficient to recover the conductivity in any subregion.

3.2. Equipotential curves as graphs. This section considers the special case in which equipotential curves are graphs in $x$, i.e., $\dot{x}(t)>0$. We show that a reparametrization reduces the system (3.7) to a second order ODE (see (3.16) below), first introduced in [23].

Since $\dot{x}>0$, the inverse function $t=g(x)$ exists; define $\psi(x)=y(g(x))$. Implicit differentiation gives

$$
\begin{aligned}
& \dot{x}(g(x))=\frac{1}{g^{\prime}(x)}, \quad \dot{y}(g(x))=\frac{\psi^{\prime}(x)}{g^{\prime}(x)}, \\
& \ddot{x}(g(x))=-\frac{g^{\prime \prime}(x)}{g^{\prime 3}(x)}, \quad \text { and }
\end{aligned}
$$




$$
\ddot{y}(g(x))=\left(\psi^{\prime \prime}(x)-\psi^{\prime}(x) \frac{g^{\prime \prime}(x)}{g^{\prime}(x)}\right) \frac{1}{g^{\prime 2}(x)} .
$$

Substitute (3.11) and (3.12) into the equation for $\ddot{x}$ in (3.7) (evaluated at $t=$ $g(x))$, and multiply both sides by $g^{\prime 2}(x)$ to obtain

$$
-\frac{g^{\prime \prime}(x)}{g^{\prime}(x)}=\frac{|J|_{x}}{|J|}(x, \psi(x))-2 \psi^{\prime}(x) \frac{|J|_{y}}{|J|}(x, \psi(x))+\psi^{\prime 2}(x) \frac{|J|_{x}}{|J|}(x, \psi(x)) .
$$

Multiply the equation for $\ddot{y}$ in (3.7) (evaluated at $t=g(x)$ ) by $g^{\prime 2}(x)$, and use (3.11) and (3.13) to obtain

$$
\begin{aligned}
\psi^{\prime \prime}(x)-\psi^{\prime}(x) \frac{g^{\prime \prime}(x)}{g^{\prime}(x)}=\frac{|J|_{y}}{|J|}(x, \psi(x)) & -2 \psi^{\prime}(x) \frac{|J|_{x}}{|J|}(x, \psi(x)) \\
& -\psi^{\prime 2}(x) \frac{|J|_{y}}{|J|}(x, \psi(x)) .
\end{aligned}
$$

Finally, use (3.14) in (3.15) to obtain the second order nonlinear equation

$$
\psi^{\prime \prime}(x)=\left(1+\psi^{\prime}(x)\right)\left(-\frac{|J|_{x}}{|J|}(x, \psi(x)) \psi^{\prime}(x)+\frac{|J|_{y}}{|J|}(x, \psi(x))\right) .
$$

The equation (3.16) was derived in [23] by different methods.

If an equipotential line $\{(x, y): u(x, y)=\lambda\}$ is a graph in $x$, then, instead of using the boundary value problem for the geodesic system (3.7), (3.9), one can solve (3.16) subject to

$$
\psi(0)=y_{0}, \quad \psi(1)=y_{1},
$$

where $\left(0, y_{0}\right),\left(1, y_{1}\right) \in \partial \Omega$ are boundary points of equal potential: $f\left(0, y_{0}\right)=f\left(1, y_{1}\right)$.

3.3. Description of the reconstruction algorithm. We present a reconstruction algorithm based on the result in Theorem 3.6. The boundary voltage potential $f$ is given at discrete points on $\Gamma \subset \partial \Omega$. A subregion $\tilde{\Omega}$ such that $\Gamma=\overline{\tilde{\Omega}} \cap \partial \Omega$ is given, without a priori knowledge of the geodesics. The interior data $|J|$, originally given on a regular grid in the subregion $\tilde{\Omega}$, is extended by (Lipschitz) continuity to the regular grid in the entire domain $\Omega$ : for example, in the numerical implementation in section $4,|J|$ is extended by continuity and constant in the $y$-direction.

The reconstruction is done in three steps. First, we reconstruct the equipotential curves joining pairs of (equipotential) points on $\Gamma$. We keep only the curves which lie entirely in the interior of $\tilde{\Omega}$ and discard the others. These curves span (and define) regions $\Omega_{\alpha, \beta}$ as in (1.4). In the numerical experiments presented in section 4 $\tilde{\Omega}$ contains only one (maximal) such $\Omega_{\alpha, \beta}$. Next we determine the voltage potential field $u$ on the regular subgrid covering the regions $\Omega_{\alpha, \beta}$, and, finally, we recover the conductivity on this subgrid.

Step 1. Reconstruction of the equipotential lines. For clarity, we describe in detail the special case when equipotential lines are graphs in $x$, and we solve the boundary value problem (3.16), (3.17). We then outline the difference in the general case (3.7), (3.9). The numerical studies in section 4 consider both cases. To further simplify the presentation, without loss of generality, we assume that $y_{0}=y_{1}=\lambda$. By introducing $\varphi(x)=\psi(x)-\lambda$, the boundary value problem (3.16), (3.17) becomes

$$
\begin{aligned}
& \varphi^{\prime \prime}(x)=F\left(x, \varphi+\lambda, \varphi^{\prime}\right), \\
& \varphi(0)=\varphi(1)=0,
\end{aligned}
$$

Copyright $@$ ㅇ by SIAM. Unauthorized reproduction of this article is prohibited. 
where

$$
F\left(x, \varphi, \varphi^{\prime}\right)=\left(1+\varphi^{\prime 2}\right)\left(-\partial_{x} \ln |J|(x, \varphi) \varphi^{\prime}+\partial_{y} \ln |J|(x, \varphi)\right) .
$$

Recall that, under our assumptions, the boundary value problem (3.18), (3.19) has a unique solution. Among the several algorithms available to solve (3.18), (3.19) we use the iterative method of Picard successive approximations for the equivalent Fredholm system:

$$
\begin{aligned}
& \varphi(x)=-\int_{0}^{1} G(x, s) F\left(s, \varphi+\lambda, \varphi^{\prime}\right) d s, \\
& \varphi^{\prime}(x)=\int_{0}^{1} H(x, s) F\left(s, \varphi+\lambda, \varphi^{\prime}\right) d s
\end{aligned}
$$

where the Green's functions $G(x, s)$ and $H(x, s)$ are given by

$$
\begin{gathered}
G(x, s)= \begin{cases}s(1-x) & \text { for } 0 \leq s \leq x \leq 1 \\
x(1-s) & \text { for } 0 \leq x \leq s \leq 1\end{cases} \\
H(x, s)= \begin{cases}s & \text { for } 0 \leq s<x \leq 1 \\
s-1 & \text { for } 0 \leq x<s \leq 1\end{cases}
\end{gathered}
$$

The system (3.21), (3.22) above is treated as a fixed point problem

$$
Y(x)=\mathbf{T}[Y](x),
$$

where the operator $\mathbf{T}$ is given by

$$
\begin{gathered}
\mathbf{T}[Y](x)=\int_{0}^{1} K(x, s) F(s, Y(s)+\mu) d s, \quad \text { with } \\
K(x, s)=\left(\begin{array}{cc}
G(x, s) & 0 \\
0 & H(x, s)
\end{array}\right), \text { and } \mu=\left(\begin{array}{c}
\lambda \\
0
\end{array}\right) .
\end{gathered}
$$

By the equivalence of (3.25) with the boundary value problem (3.16), (3.17), we already know that the operator $\mathbf{T}$ in $(3.25)$ has a unique fixed point in $C\left([0,1] ; \mathbb{R}^{2}\right)$. We solve (3.25) iteratively by the successive approximations $Y_{k}(x)=\mathbf{T} Y_{k-1}(x)$, $(k=0,1,2, \ldots)$ on the grid $\omega_{h}=\left\{x_{k}=k h, 1=h(M+1), k=0,1,2, \ldots, M+1\right\}$. This method is known to be slow to converge. However, a sufficiently close initial guess speeds up the convergence. The specific procedure of choosing the initial approximation is discussed in section 4.2.1. As a stopping criterion we use the relative 2-norm estimate

$$
\frac{\left\|Y_{k}-Y_{k-1}\right\|}{\left\|Y_{k}\right\|} \leq \varepsilon
$$

where $\varepsilon>0$ is a small number to be specified later.

In the general case, we solve numerically the boundary value problem (3.7), (3.9) by a similar method. The system of the second order differential equations is reduced to an operator equation similar to (3.25). The vector valued map $Y(x)$ now has four components, and the Green's function valued matrix $K(x, s)$ is given by

$$
K(x, s)=\left(\begin{array}{cccc}
G(x, s) & 0 & 0 & 0 \\
0 & H(x, s) & 0 & 0 \\
0 & 0 & G(x, s) & 0 \\
0 & 0 & & H(x, s)
\end{array}\right)
$$

Copyright (c) by SIAM. Unauthorized reproduction of this article is prohibited. 
with $G$ and $H$ as defined previously in (3.23), respectively, (3.24).

Step 2. Determining the voltage potential field. By solving numerically the two point boundary value problems for pairs of equipotential boundary points in $\Gamma$ and collecting only the curves which lie entirely in $\tilde{\Omega}$, we obtain a finite set of equipotential lines spanning (and defining) the regions $\Omega_{\alpha, \beta}$ as in (1.4). As a result, a finite set of triples $\left(x_{m}, y_{m}, u_{m}\right),\left(m=1,2, \ldots, m^{*}\right)$ is found, where the planar set of points $\left(x_{m}, y_{m}\right)$ distributed in this region represents the equipotential lines. Our goal is to determine the voltage potential $u\left(x_{i}, y_{j}\right)$ on the initial grid (on which the interior data $\left|J\left(x_{i}, y_{j}\right)\right|$ is given). We first interpolate over the triangulated partition of each such $\Omega_{\alpha, \beta}$, with points $\left(x_{m}, y_{m}\right)$ being the vertices of nonoverlapping triangles $T_{1}, \ldots, T_{s}$. Then, for every vector $u_{1}, \ldots, u_{m}, \ldots, u_{m^{*}}$, we construct the voltage potential in each $\Omega_{\alpha, \beta}$ as an interpolating spline over the triangulated partition, i.e., a polynomial of the specified degree in every triangle $T_{1}, \ldots, T_{s}$ which matches $u_{1}, \ldots, u_{m}, \ldots, u_{m^{*}}$ at the corresponding vertices. We employ both the bicubic and biquintic spline interpolations (see, e.g., [7], [5]).

Step 3. Reconstruction of the conductivity. Once the voltage potential field $u\left(x_{i}, y_{j}\right)$ is determined on the regular grid, we approximate the magnitude of the electric field $|\nabla u|_{i j}$ on the grid by

$$
|\nabla u|_{i j}=\frac{1}{2 h_{x} h_{y}} \sqrt{\left(h_{y}\left(u\left(x_{i+1}, y_{j}\right)-u\left(x_{i-1}, y_{j}\right)\right)\right)^{2}+\left(h_{x}\left(u\left(x_{i}, y_{j+1}\right)-u\left(x_{i}, y_{j-1}\right)\right)\right)^{2}}
$$

and recover the conductivity on the grid by

$$
\sigma\left(x_{i}, y_{j}\right)=\frac{\left|J\left(x_{i}, y_{j}\right)\right|}{\left|\nabla u\left(x_{i}, y_{j}\right)\right|} .
$$

4. Numerical study. We present two numerical experiments of the reconstruction algorithm above. In both experiments, the domain is the unit square: $\Omega=$ $(0,1) \times(0,1)$.

4.1. Data simulation. To generate the admissible pairs $(f,|J|)$ we first solve numerically the Dirichlet problem (1.1). In both experiments we use the almost twoto-one boundary condition $f$ given by $f(x, 0)=0, f(x, 1)=1, f(0, y)=f(1, y)=y$, but two different models of conductivity distributions are considered.

The first choice is the four modes function

$$
\sigma(x, y)=1+\sigma_{s}(x, y)
$$

where $\sigma_{s}(x, y)=0.3 \cdot(A(x, y)-B(x, y)-C(x, y))$ with

$$
\begin{aligned}
& A=0.3 \cdot(1-3(2 x-1))^{2} \cdot e^{-9 \cdot(2 x-1)^{2}-(6 y-2)^{2}}, \\
& B=\left[\frac{3(2 x-1)}{5}-27 \cdot(2 x-1)^{3}-(3 \cdot(2 y-1))^{5}\right] \cdot e^{-\left(9 \cdot(2 x-1)^{2}+9 \cdot(2 y-1)^{2}\right)}, \\
& C=e^{-(3 \cdot(2 x-1)+1)^{2}-9 \cdot(2 y-1)^{2}}
\end{aligned}
$$

see the left graph in Figure 4.1.

The second choice of conductivity uses a piecewise-constant function modeled on the CT image of a human torso; see Figure 4.2. More precisely, we scale the density distribution in the original $\mathrm{CT}$ image to a conductivity distribution ranging from 1 to $1.8 \mathrm{~S} / \mathrm{m}$. 

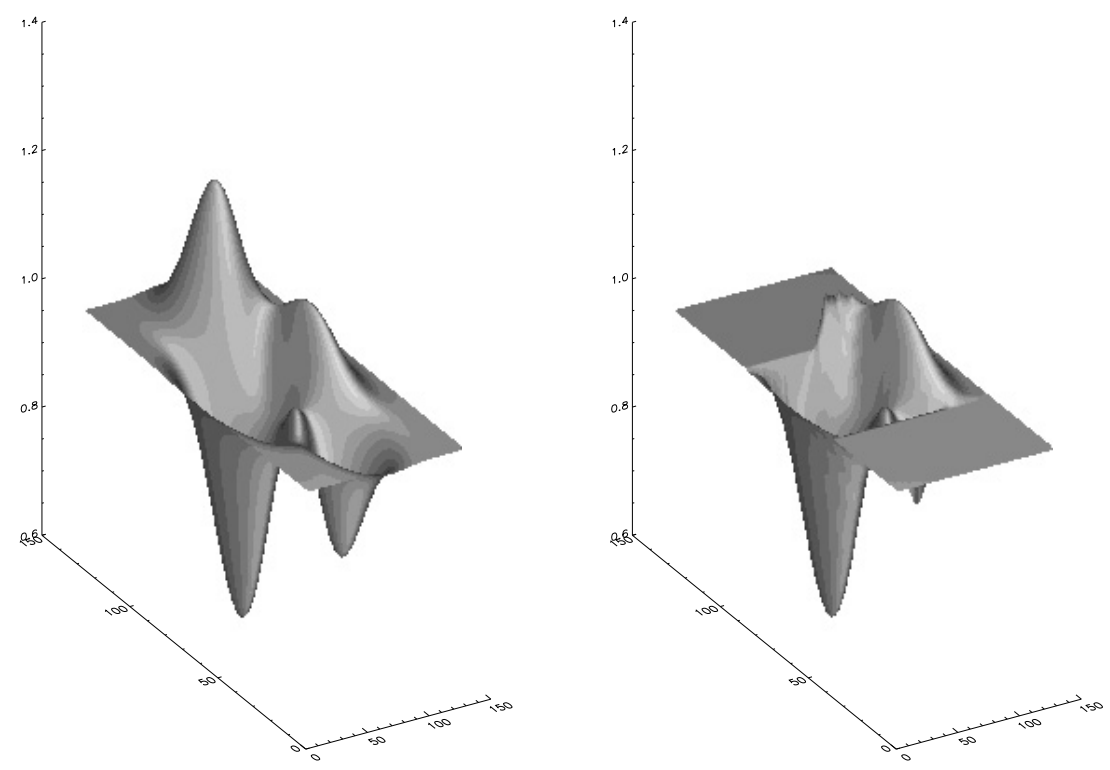

FIG. 4.1. Original (left) and reconstructed (right) conductivity distributions in the broad strip. No noise was added in the interior data.

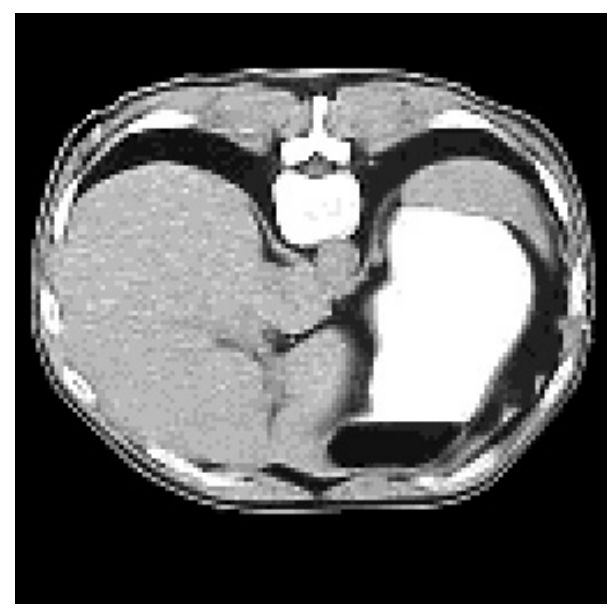

FIG. 4.2. The second choice of conductivity to be recovered.

To solve (1.1), we seek its solution in the form $u=u_{h}+v$, where $u_{h}(x, y)=y$ is the harmonic function with trace $f$ on $\partial \Omega$, and solve for $v$ in the Dirichlet problem for the Poisson equation

$$
\nabla \cdot(\sigma \nabla v)=-\frac{\partial \sigma}{\partial y},\left.\quad v\right|_{\partial \Omega}=0 .
$$

For the latter problem we use a longitudinal-transverse finite difference scheme with an optimal reordered set of Chebyshev's parameters as in [26]. We found that this method provides better accuracy than the commonly used preconditioned conjugate gradient 


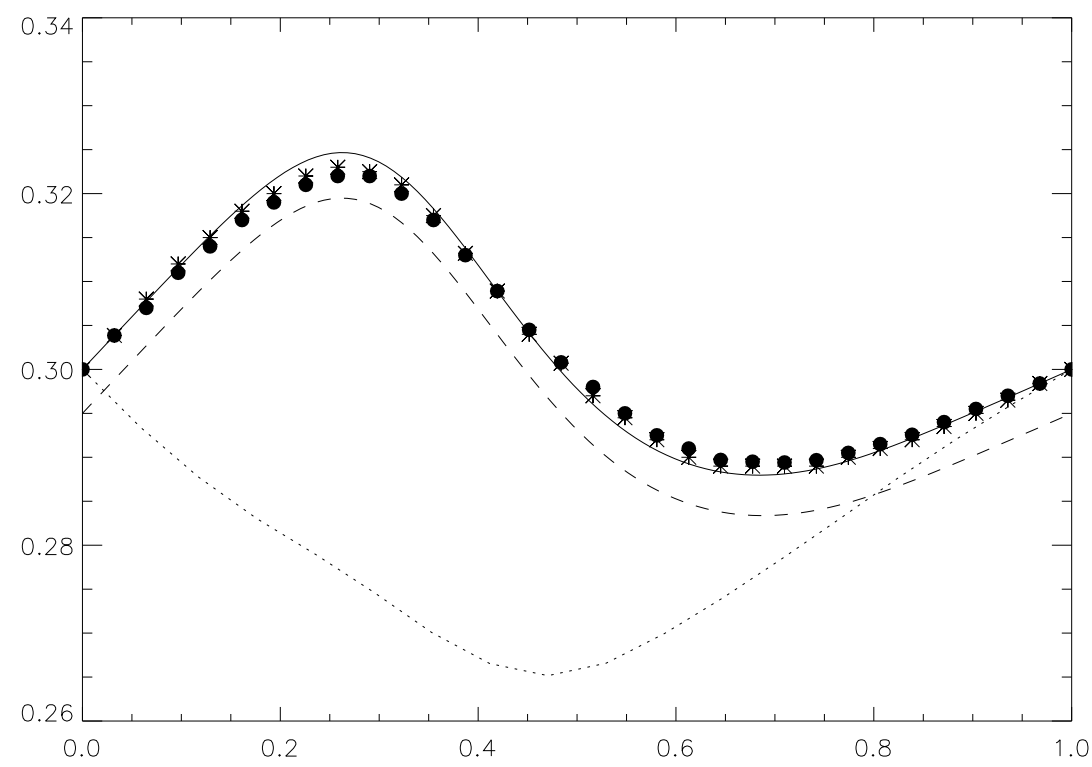

FIG. 4.3. The equipotential lines: solid $=$ original; dotted $=$ reconstructed with initial guess a horizontal segment; bullets $=$ reconstructed with initial guess the previous equipotential line via one equation; asterisks $=$ reconstructed with initial guess the previous equipotential line via the geodesic system; dashed line $=$ the previous equipotential line.

method. When computing $\nabla v$, we use the three point Lagrangian interpolation for the numerical differentiation. Once $\nabla u$ is determined, the interior data $|J|=\sigma|\nabla u|$ is computed on a regular grid in $\Omega$. In order to have a small interpolation error in the second step of the algorithm, we choose a sufficiently dense grid of $128 \times 128$ points in $\bar{\Omega}$.

Note that, in the reconstruction steps, we use only the data at points on the grid within $\tilde{\Omega}$, the immediate adjacent neighbors, and boundary grid points in $\Gamma$.

4.2. Numerical results of the reconstruction. In this section we demonstrate the computational feasibility of the reconstruction algorithm by performing some numerical experiments with the two models of the conductivity distribution described above.

4.2.1. Reconstruction of a single equipotential line. We test the first step of the algorithm in the general case that involves solving (3.7) and (3.9), as well as in the specific case that involves solving (3.16) and (3.17). We first consider the interior data generated by the smooth four modes conductivity in (4.1). We found that the Picard successive approximations method used to solve either of the boundary value problems is very sensitive to the first guess. Figure 4.3 shows the reconstruction of the equipotential line $u=0.3$ (depicted by a solid line) in the general case (bullets) and in the special case (stars) when the Picard iterations start with a special guess as detailed below. However, the dotted curve (far from the exact solid line) represents an iterate (of order comparable to the other two) when the initial guess is the horizontal segment at $y=0.3$.

Next we describe in detail how the initial guess for the Picard's iterative scheme is chosen. We use the fact, also appearing in the proof of Theorem 3.4, that the 
geodesics connecting equipotential points at the boundary are also level curves of a smooth function, free of singular points. Consequently, sufficiently close level curves have almost parallel tangents at the boundary. We use the directional information of the previously computed curve to solve an initial value problem for the geodesic system and use this solution as a first guess. For example, the tangent at the boundary point $(0,0.295)$ of the calculated equipotential line $u=0.295$ (shown in Figure 4.3 as the dashed line) is used to compute the initial guess for the equipotential line $u=0.3$ (solid line in Figure 4.3).

To describe our procedure in detail, we consider the special case of solving (3.18) subject to (3.19). Assume that the equipotential line $x \mapsto\left(x, \psi^{k}(x)\right)$ starting at the boundary grid point $\left(0, y_{k}\right) \in \Gamma$ is already computed. To find an initial guess for the neighbor equipotential line $x \mapsto\left(x, \psi^{k+1}(x)\right)$ that passes through the grid point $\left(0, y_{k+1}\right) \in \Gamma$, we solve the Cauchy problem for (3.18) subject to the initial data $\psi(0)=y_{k+1}, \psi_{x}(0)=\psi_{x}^{k}(0)$. Numerically, we solve the equivalent integral equation for the map $\varphi(x)=\psi(x)-y_{k+1}$ :

$$
\varphi(x)=\int_{0}^{x}\left[\left(\psi_{x}^{k}(0)-y_{k+1}\right) e^{\mu s}+\int_{0}^{s} e^{-\mu(t-s)}\left[F\left(t, \varphi+y_{k+1}, \varphi^{\prime}\right)-\mu \varphi(t)\right] d t\right] d s,
$$

where $F\left(x, \varphi, \varphi^{\prime}\right)$ is given by $(3.20)$.

The multiplier $e^{-\mu x}$ is the weight function in Bielecki's norm

$$
\|u\|_{\mu}=\max _{x \in[0,1]} e^{-\mu x}|u(x)|
$$

introduced to ensure the contraction property of the integral operator generated by the right-hand side of (4.2). Specifically, if $L(F)$ is a Lipschitz constant of $F$ with respect to the $\varphi$-variable, choosing $\mu>L(F)$ yields convergence and stability of Picard's iterations in the space of continuous maps on $[0,1]$.

The method above requires us to construct a first equipotential line. If the interior data $|J|$ is known in a region of constant conductivity near the boundary arc of maximum (or minimum) voltage at the boundary, then the arc itself is such an equipotential line. Otherwise the knowledge of the current at just one (arbitrarily fixed) point on $\Gamma$ suffices. If such information is not available, we need to solve the two point boundary value problem by a different numerical scheme.

The rates of convergence for both problems are good: from 4 to 15 iterations depending on the specific equipotential line $u=\lambda$ and on the stopping criterion. For instance, for the four mode conductivity model on the $128 \times 128$ grid, to compute the equipotential line $u=0.3$ with a relative error of $\epsilon \leq 10^{-5}$ in (3.26), the elapsed CPU time (on a Dell workstation) is about $17.5 \mathrm{~s}$.

4.2.2. Local reconstruction of a smooth conductivity distribution. Here we show how to reconstruct the smooth conductivity (as described in Step 3 of section 3.3) in the subregions $\Omega_{1 / 2,3 / 4}$ and $\Omega_{1 / 4,3 / 4}$ calculated above. Figure 4.4 shows the reconstructed equipotential lines and triangulated area spanned by these lines. Due to the presence of deflection points in the level lines, there are regions in the strips that are not spanned by geodesics.

Taking into account the smallness of such regions (compared to the triangulated area) and regularity of the voltage potential, we use an interpolating bicubic and biquintic spline constructed over the triangulation for extrapolating the voltage potential field into these regions. The reconstructed conductivity distributions are shown in Figures 4.1 and 4.5 compared with the original conductivity. 

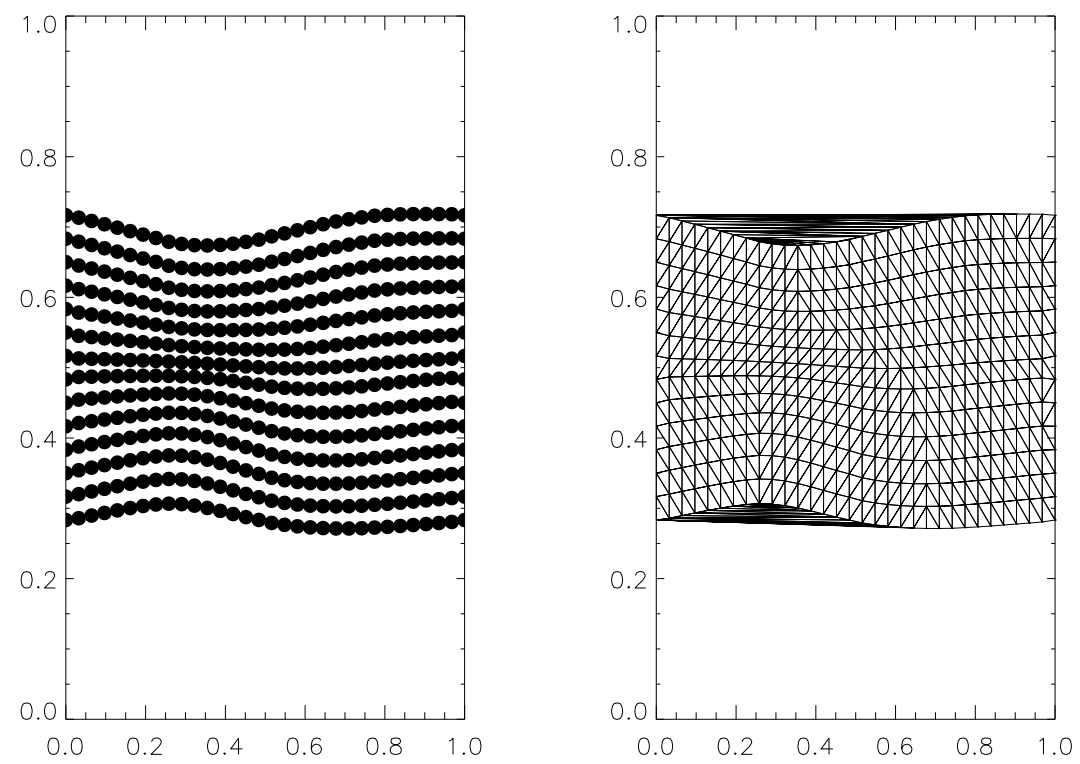

FIG. 4.4. Equipotential lines (left) and the triangulated area (right) spanned by these lines.
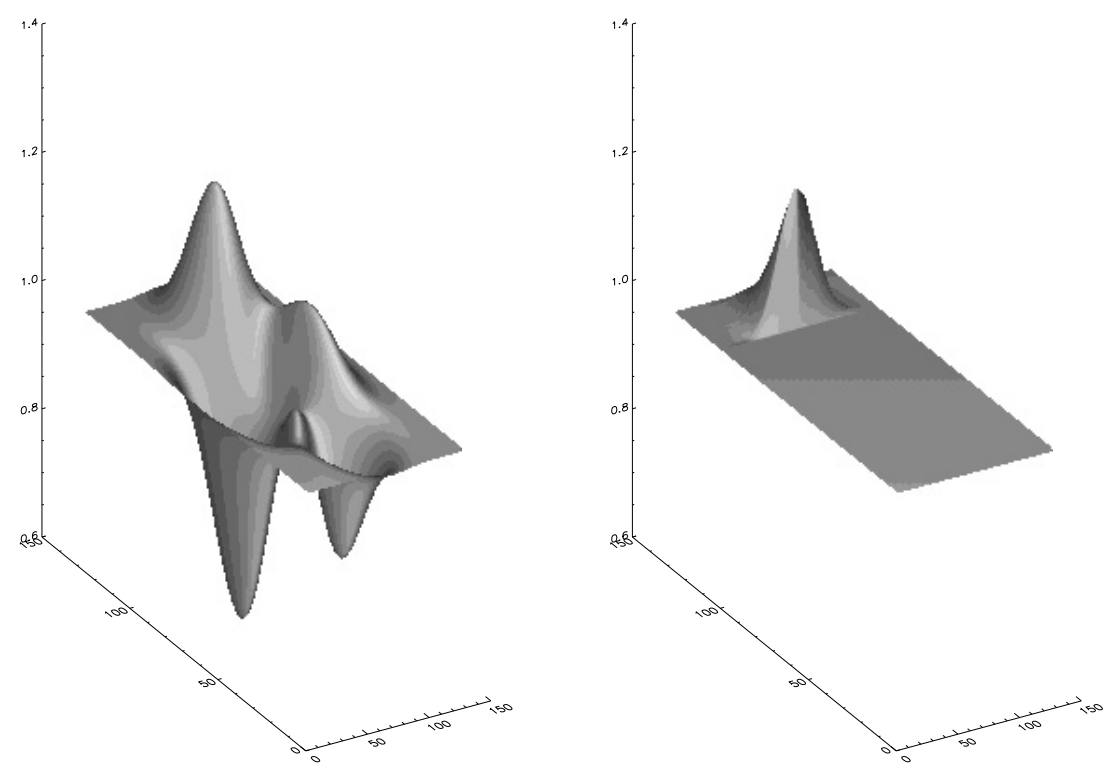

FIG. 4.5. The original (left) and reconstructed (right) conductivity in the thin strip. No noise was added in the interior data.

4.2.3. Local reconstruction of a discontinuous conductivity distribution. In this case, the equipotential lines span the entire strips as shown in Figure 4.6. Therefore, there is no need for the extrapolation. The results of reconstruction of conductivity from the noiseless interior data in both the broad and thin strips are shown in Figure 4.7. 

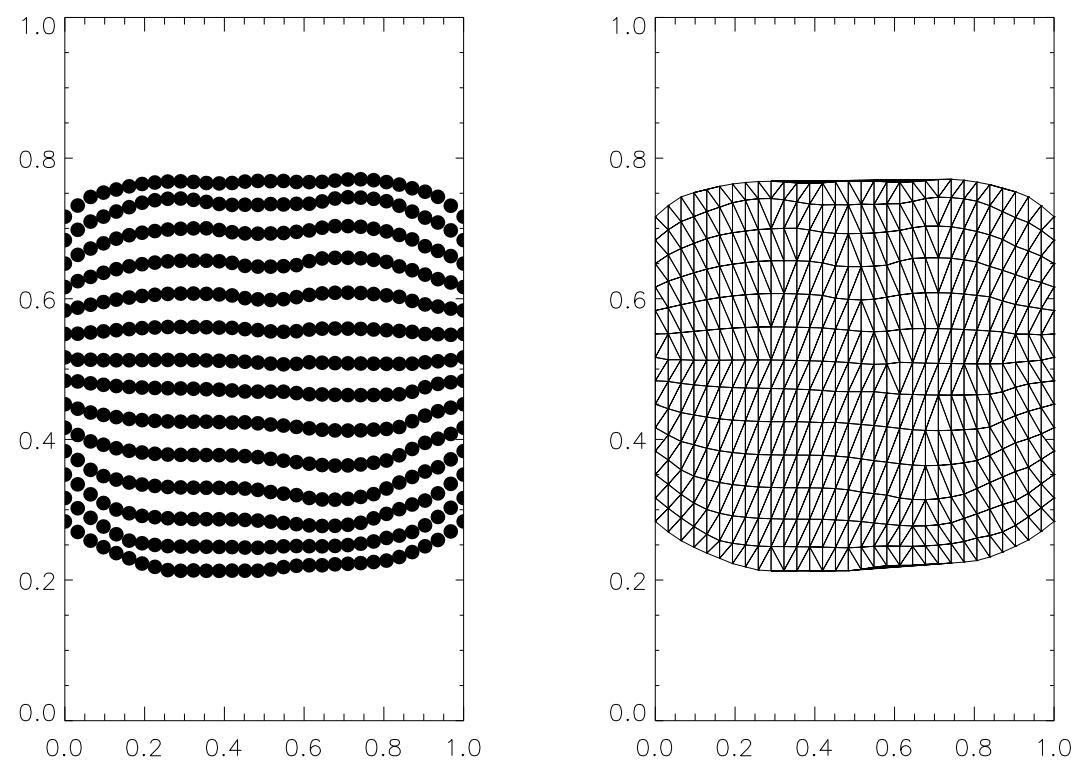

FIG. 4.6. Equipotential lines (left) and the triangulated area (right) spanned by these lines.

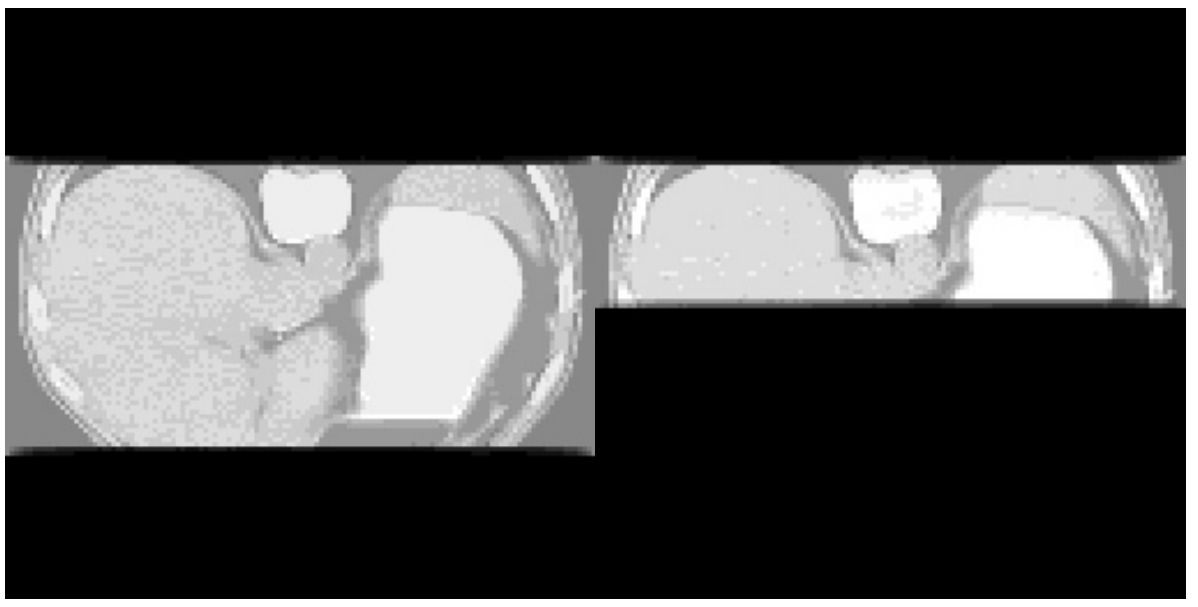

FIG. 4.7. Reconstructed conductivity from noiseless interior data: broad (left) and thin (right) strips.

Comparison of the original image shown in Figure 4.2 with the reconstructed one shows that they are not identical. This is due to both the algorithmic and roundoff errors, whose total estimated level does not exceed $10^{-4}$.

To investigate numerically the stability of the proposed algorithm, we model the perturbed interior data $|\tilde{J}|$ by adding the Gaussian random matrix $\xi$ with a mean of zero and a specified standard deviation to the discrete function $|\hat{J}|$, i.e.,

$$
|\tilde{J}|=|\hat{J}|+\xi
$$

The realizations of the perturbed interior data are then used in the reconstruction 


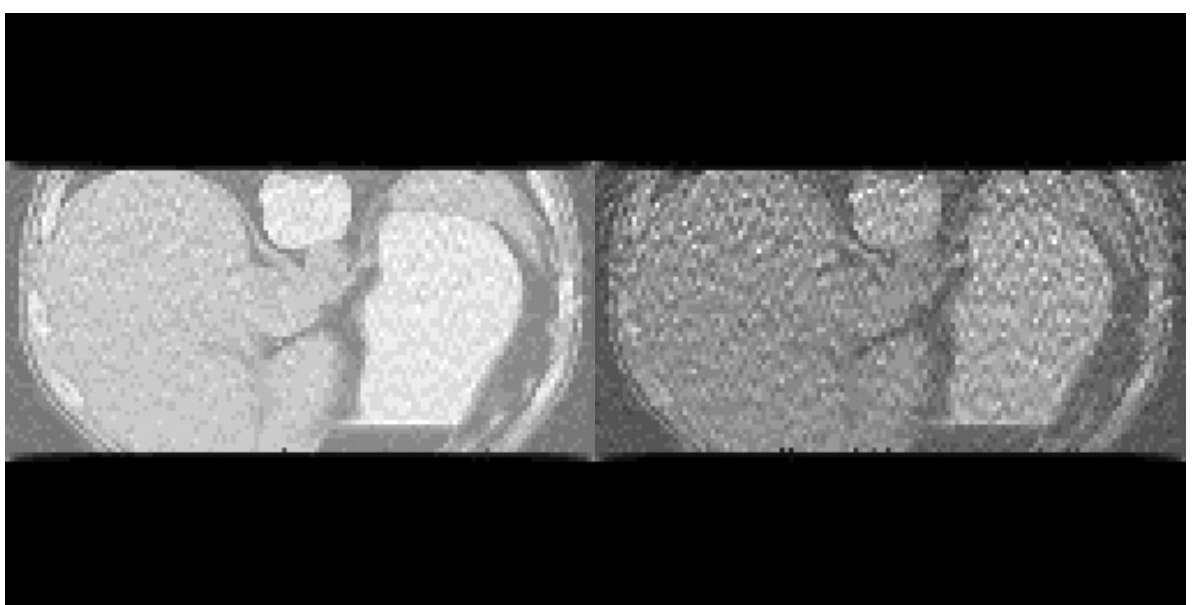
(right).

FIG. 4.8. Reconstructed conductivity in the broad strip from noisy data of $1 \%$ (left) and $5 \%$

algorithm. Figure 4.8 shows the mean images obtained from samples containing 20 realizations of the random matrix $\xi$. The values of the standard deviation of $\xi$ are chosen to provide the noise levels at about $1 \%$ and $5 \%$, correspondingly.

5. Conclusions. We considered the conductivity imaging problem from interior knowledge of the magnitude of one current density field $|J|$. In any dimension and for general boundary voltages, we showed that equipotential sets are area minimizing with respect to a conformal metric determined by $|J|$. In two dimensions, we identified a class of boundary voltages (almost two-to-one) for which the minimization problem has a unique solution: there is a unique geodesic joining pairs of equipotential boundary points.

For this two dimensional problem, we give a reconstruction method based on the calculation of geodesics joining equipotential boundary points. If the imposed boundary voltage is known only on a subset $\Gamma$ of $\partial \Omega$ which contains pairs of equipotential points, this calculation can be localized to the equipotential lines joining those points. Moreover, if $|J|$ is measured only in a subregion $\tilde{\Omega}$ with $\tilde{\Omega} \cap \partial \Omega=\Gamma$, our method recovers the conductivity in the subdomain spanned by the equipotential lines entirely embedded in $\tilde{\Omega}$. The method also decides from the data whether or not $\tilde{\Omega}$ contains such equipotential curves.

In the numerical algorithm, the boundary value problem has been solved by an iterative procedure in which each previously calculated equipotential line has been used as the initial approximation for the next one. The algorithms performed well even with rough data and in the presence of noise.

Appendix. Calculating a covariant derivative. For the reader's convenience, we include the calculation of the covariant derivative of the unit tangent field to the level curves of $u$ in the metric $g=a^{2} I$. Throughout this appendix $|\cdot|$ stands for the Euclidean norm, whereas $|\cdot|_{g}$ and $\langle\cdot, \cdot\rangle_{g}$ stand for the norm and, respectively, scalar product in the metric $g=a^{2} I$.

Since $u \in C^{2}(\Omega)$ and $|\nabla u|>0$, the level sets are $C^{2}$-smooth curves. Let $\left\{\delta_{x}, \delta_{y}\right\}$ be the coordinate frame in the tangent bundle $T \Omega$ dual to the $\{x, y\}$-coordinates. In these coordinates, the unit tangent $\tau$ and the unit normal $n$ (with respect to the 
$g$-metric) are, respectively,

$$
\begin{gathered}
\tau=-\frac{1}{a} \frac{u_{y}}{|\nabla u|} \delta_{x}+\frac{1}{a} \frac{u_{x}}{|\nabla u|} \delta_{y}, \\
n=\frac{1}{a} \frac{u_{x}}{|\nabla u|} \delta_{x}+\frac{1}{a} \frac{u_{y}}{|\nabla u|} \delta_{y} .
\end{gathered}
$$

Using the formulae for Christoffel's symbols in (3.8), the following covariant derivative formulas hold:

$$
\nabla_{\delta_{x}} \delta_{x}=\frac{a_{x}}{a} \delta_{x}-\frac{a_{y}}{a} \delta_{y}, \quad \nabla_{\delta_{x}} \delta_{y}=\frac{a_{y}}{a} \delta_{x}+\frac{a_{x}}{a} \delta_{y}, \quad \nabla_{\delta_{y}} \delta_{y}=\frac{-a_{x}}{a} \delta_{x}+\frac{a_{y}}{a} \delta_{y} .
$$

Let $D_{t} \tau$ be the covariant derivative along the level curve. Since $|\tau|_{g}=1$, we have that $D_{t} \tau$ is orthogonal to $\tau$ and the signed curvature is the projection on $n$ : $k=\left\langle D_{t} \tau, n\right\rangle_{g}$.

We compute the covariant derivative of $\tau$ in (A.1) along a level curve. Subscripts denote the differentiation along the coordinates:

$$
\begin{aligned}
D_{t} \tau= & -\frac{u_{y}}{a|\nabla u|} \nabla_{\delta_{x}} \tau+\frac{u_{x}}{a|\nabla u|} \nabla_{\delta_{y}} \tau \\
= & -\frac{u_{y}}{a|\nabla u|}\left(\frac{u_{y}}{|a \nabla u|}\right)_{x} \delta_{x}+\frac{u_{y}^{2}}{a^{2}|\nabla u|^{2}} \nabla_{\delta_{x}} \delta_{x} \\
& -\frac{u_{y}}{a|\nabla u|}\left(\frac{u_{x}}{a|\nabla u|}\right)_{x} \delta_{y}-\frac{u_{y} u_{x}}{a^{2}|\nabla u|^{2}} \nabla_{\delta_{x}} \delta_{y} \\
& -\frac{u_{x}}{a|\nabla u|}\left(\frac{u_{y}}{a|\nabla u|}\right)_{y} \delta_{x}-\frac{u_{x} u_{y}}{a^{2}|\nabla u|^{2}} \nabla_{\delta_{y}} \delta_{x} \\
& +\frac{u_{x}}{a|\nabla u|}\left(\frac{u_{x}}{a|\nabla u|}\right)_{y} \delta_{y}+\frac{u_{x}^{2}}{a^{2}|\nabla u|^{2}} \nabla_{\delta_{y}} \delta_{y} .
\end{aligned}
$$

By replacing the covariant derivatives (A.3) in the formula above, after cancellation, we obtain

$$
\begin{aligned}
D_{t} \tau=\frac{1}{a^{2}}\{ & {\left[\frac{u_{y}}{|\nabla u|}\left(\frac{u_{y}}{|\nabla u|}\right)_{x}-\frac{a_{y} u_{x} u_{y}}{a|\nabla u|^{2}}-\frac{u_{x}}{|\nabla u|}\left(\frac{u_{y}}{|\nabla u|}\right)_{y}-\frac{a_{x} u_{x}^{2}}{a|\nabla u|^{2}}\right] \delta_{x} } \\
+ & {\left.\left[-\frac{a_{y} u_{y}^{2}}{a|\nabla u|^{2}}-\frac{u_{y}}{|\nabla u|}\left(\frac{u_{x}}{|\nabla u|}\right)_{x}-\frac{a_{x} u_{x} u_{y}}{a|\nabla u|^{2}}+\frac{u_{x}}{|\nabla u|}\left(\frac{u_{x}}{|\nabla u|}\right)_{y}\right] \delta_{y}\right\} . }
\end{aligned}
$$

The curvature $k=\left\langle D_{t} \tau, n\right\rangle$, with $n$ in (A.2), is then given by

$$
\begin{aligned}
k=\frac{1}{a}\left\{\frac{u_{y} u_{x}}{|\nabla u|^{2}}\left(\frac{u_{y}}{|\nabla u|}\right)_{x}-\frac{a_{y} u_{x}^{2} u_{y}}{a|\nabla u|^{3}}-\frac{u_{x}^{2}}{|\nabla u|^{2}}\left(\frac{u_{y}}{|\nabla u|}\right)_{y}-\frac{a_{x} u_{x}^{3}}{a|\nabla u|^{3}}\right. \\
\left.\quad-\frac{u_{y}^{2}}{|\nabla u|^{2}}\left(\frac{u_{x}}{|\nabla u|}\right)_{x}-\frac{a_{y} u_{y}^{3}}{a|\nabla u|^{3}}+\frac{u_{x} u_{y}}{|\nabla u|^{2}}\left(\frac{u_{x}}{|\nabla u|_{y}}\right)_{y}-\frac{a_{x} u_{x} u_{y}^{2}}{a|\nabla u|^{3}}\right\} .
\end{aligned}
$$

The following identity follows from straightforward differentiation:

$$
u_{y} u_{x}\left(\frac{u_{y}}{|\nabla u|}\right)_{x}+u_{x} u_{y}\left(\frac{u_{x}}{|\nabla u|}\right)_{y}+u_{x}^{2}\left(\frac{u_{x}}{|\nabla u|}\right)_{x}+u_{y}^{2}\left(\frac{u_{y}}{|\nabla u|}\right)_{y}=0
$$

Copyright $\odot$ by SIAM. Unauthorized reproduction of this article is prohibited. 
By adding and subtracting two terms, and then dividing by $|\nabla u|^{2}$, the identity (A.7) implies that

$$
\begin{gathered}
\frac{u_{y} u_{x}}{|\nabla u|^{2}}\left(\frac{u_{y}}{|\nabla u|}\right)_{x}+\frac{u_{x} u_{y}}{|\nabla u|^{2}}\left(\frac{u_{x}}{|\nabla u|}\right)_{y}-\frac{u_{x}^{2}}{|\nabla u|^{2}}\left(\frac{u_{y}}{|\nabla u|}\right)_{y}-\frac{u_{y}^{2}}{|\nabla u|^{2}}\left(\frac{u_{x}}{|\nabla u|}\right)_{x} \\
=-\left(\frac{u_{x}}{|\nabla u|}\right)_{x}-\left(\frac{u_{y}}{|\nabla u|}\right)_{y}
\end{gathered}
$$

Substituting (A.8) into (A.6), we obtain the curvature formula (3.2).

Acknowledgments. The authors thank the referees for their helpful criticisms. The research in this paper originated in authors' discussions during the BIRS workshop "Inverse Problems: Recent Progress and New Challenges" (08w5065), Nov 16-21, 2008. We would like to thank the Banff International Research Station for the wonderful hospitality.

\section{REFERENCES}

[1] G. Alessandrini, An identification problem for an elliptic equation in two variables, Ann. Mat. Pura Appl. (4), 145 (1986), pp. 265-295.

[2] H. Ammari, Y. CapdeboscQ, H. Kang, and A. Kozhemiak, Mathematical models and reconstruction methods in magneto-acoustic imaging, European J. Appl. Math., 20 (2009), pp. 303-317.

[3] H. Ammari, E. Bonnetier, Y. CapdeboscQ, M. Tanter, and M. Fink, Electrical impedance tomography by elastic deformation, SIAM J. Appl. Math., 68 (2008), pp. 1557-1573.

[4] H. Ammari AND H. Kang, Multi-scale and multi-physics biomedical imaging modalities, in Handbook of Mathematical Methods in Imaging, O. Scherzer, ed., Springer, New York, to appear.

[5] P. M. Anselon and P. J. Laurent, A general method for construction of interpolating and smoothing spline-functions, Numer. Math., 12 (1968), pp. 66-191.

[6] G. Bal And J. C. Schotland, Inverse scattering and acousto-optic imaging, Phys. Rev. Lett., 104 (2010), 043902.

[7] C. DE Boor, Bicubic spline interpolation, J. Math. and Phys., 41 (1962), pp. 212-218.

[8] E. Bombieri, E. De Giongi, And E. Giusti, Minimal cones and the Bernstein problem, Invent. Math., 7 (1969), pp. 243-268.

[9] D. ISAACSON AND M. CHENEY, Effects of measurement precision and finite numbers of electrodes on linear impedance imaging algorithms, SIAM J. Appl. Math., 51 (1991), pp. 1705-1731.

[10] B. Gebauer and O. Scherzer, Impedance-acoustic tomography, SIAM J. Appl. Math., 69 (2008), pp. 565-576.

[11] D. Gilbarg and N. S. Trudinger, Elliptic Partial Differential Equations, 2nd ed., SpringerVerlag, New York, 2001.

[12] H. Federer, Curvature measures, Trans. Amer. Math. Soc., 62 (1959), pp. 418-491.

[13] M. J. Joy, A. I. Nachman, K. F. Hasanov, R. S. Yoon, and A. W. Ma, A new approach to current density impedance imaging (CDII), in Proceedings of the 12th Annual Meeting of the International Society for Magnetic Resonance in Medicine (ISMRM), Kyoto, Japan, 2004, CD-ROM, abstract no. 356.

[14] S. KIm, O. Kwon, J. K. SEO, And J.-R. Yoon, On a nonlinear partial differential equation arising in magnetic resonance electrical impedance tomography, SIAM J. Math. Anal., 34 (2002), pp. 511-526.

[15] O. Kwon, E. J. Woo, J. R. Yoon, And J. K. Seo, Magnetic resonance electric impedance tomography (MREIT): Simulation study of J-substitution algorithm, IEEE Trans. Biomed. Eng., 49 (2002), pp. 160-167.

[16] O. Kwon, J. Y. Lee, And J. R. Yoon, Equipotential line method for magnetic resonance electrical impedance tomography, Inverse Problems, 18 (2002), pp. 1089-1100.

[17] J. M. LeE, Riemannian Manifolds, Springer, New York, 1997.

[18] J. Y. LEE, A reconstruction formula and uniqueness of conductivity in MREIT using two internal current distributions, Inverse Problems, 20 (2004), pp. 847-858.

[19] J. J. Liu, J. K. Seo, M. Sini, And E. J. Woo, On the conductivity imaging by MREIT: Available resolution and noisy effect, J. Phys. Conf. Ser., 73 (2007), paper 012013. 
[20] J. J. Liu, J. K. Seo, M. Sini, And E. J. Woo, On the convergence of the harmonic $B^{z}$ algorithm in magnetic resonance electrical impedance tomography, SIAM J. Appl. Math., 67 (2007), pp. 1259-1282.

[21] Q. MA AND B. HE, Investigation on magnetoacoustic signal generation with magnetic induction and application to electrical conductivity reconstruction, Phys. Med. Biol., 52 (2007), pp. 5085-5099.

[22] N. Mandache, Exponential instability in an inverse problem for the Schrödinger equation, Inverse Problems, 17 (2001), pp. 1435-1444.

[23] A. Nachman, A. Tamasan, and A. Timonov, Conductivity imaging with a single measurement of boundary and interior data, Inverse Problems, 23 (2007), pp. 2551-2563.

[24] A. Nachman, A. Tamasan, and A. Timonov, Recovering the conductivity from a single measurement of interior data, Inverse Problems, 25 (2009), article 035014.

[25] M. Z. NAShed AND A. TAMASAn, Structural stability in a minimization problem and applications to conductivity imaging, Inverse Probl. Imaging, to appear.

[26] A. A. Samarskit, The Theory of Difference Schemes, Marcell Decker, New York, 2001.

[27] G. C. Scott, M. L. Joy, R. L. Armstrong, and R. M. Henkelman, Measurement of nonuniform current density by magnetic resonance, IEEE Trans. Med. Imag., 10 (1991), pp. $362-374$.

[28] E. J. Woo And J. K. SeO, Magnetic resonance electrical impedance tomography (MREIT) for high resolution conductivity imaging, Physiol. Meas., 29 (2008), pp. R1-R26.

[29] X. LI, Y. XU, AND B. He, Imaging electrical impedance from acoustic measurements by means of magnetoacoustic tomography with magnetic induction (MAT-MI), IEEE Trans. Biomed. Eng., 54 (2007), pp. 323-330.

[30] N. Zhang, Electrical Impedance Tomography Based on Current Density Imaging, M.Sc. thesis, University of Toronto, Ontario, Canada, 1992.

[31] W. P. Ziemer, Weakly Differentiable Functions, Springer, New York, 1069.

Copyright (c) by SIAM. Unauthorized reproduction of this article is prohibited. 